\title{
NEW INSIGHTS INTO TIME SYNCHRONIZATION OF MIMO SYSTEMS WITH INTERFERENCE
}

\author{
S. Hiltunen ${ }^{(1),(2),(3)} \quad$ P. Chevalier ${ }^{(3),(1)} \quad$ P.Loubaton $^{(2)}$ \\ (1) Thales-Communications-Security, AMS/TCP, 4 Av. des Louvresses, 92622 Gennevilliers Cédex, France \\ (2) Univ. Paris-Est Marne-la-Vallée, LIGM, UMR CNRS 8049, 5 Bd. Descartes, 77454 Marne la Vallée Cédex 2, France \\ (3) CNAM, Laboratoire CEDRIC, 292 rue Saint-Martin, 75141 Paris Cédex 3, France.
}

\begin{abstract}
This paper concerns time synchronization of MIMO systems. The current most powerful receiver is a generalized likelihood ratio test (GLRT) receiver, and assumes unknown, stationary, circular, and spatially colored Gaussian noise. However, this receiver is more complex than its non-GLRT counterparts, which, unfortunately, do not perform as well in most cases. As the complexity is an important issue for practical implementations and may be prohibitive for a large number of antennas, the purpose of this paper is to propose several ways of decreasing the complexity of the GLRT receiver while keeping its performance. Simplifications of the GLRT receiver and optimization of parameters, jointly with new low-complexity receivers, are introduced. The performance of the new receivers is analyzed and compared with that of the GLRT receiver, enlightening the practical interest of these receivers.
\end{abstract}

Index Terms - Time Synchronization, MIMO, Single Carrier, GLRT, Interference.

\section{INTRODUCTION}

Time and frequency synchronization of MIMO systems have been strongly studied in the last fifteen years. Both coarse and fine time synchronization jointly with frequency offset estimation and compensation have been deeply analyzed and many techniques have been proposed either for timefrequency synchronization $[1,2]$ or time synchronization only [3-7]. Nevertheless, most of these techniques assume an absence of interference. The scarce papers of the literature dealing with MIMO synchronization in the presence of interference correspond to [1,5-7]. However, [1] and [5] only consider the problem of MIMO synchronization in the presence of multi-users interference (MUI), and [6] seems to be the only paper dealing with MIMO synchronization in the presence of interference of any kind, such as hostile jammers. In [6], several receivers are proposed for time synchronization for both flat fading and frequency selective fading channels. But for complexity reasons, only those developed for flat fading channels seem to be realistic for practical situations. Note however that these receivers may also be used for frequency selective channels, considering the secondary propagation multi-paths as interference. In [6], two receivers for flat fading channels, robust to interferences, are derived from an MMSE and a GLRT approach respectively. The GLRT receiver, called GLRT2 receiver in the following, assumes unknown, stationary, Gaussian, spatially colored and temporally white total noise, contrary to the GLRT1 receiver which assumes stationary, Gaussian and spatially white noise. The GLRT2 receiver has been shown in [6] to perform better than the others in the presence of interference for non-orthogonal synchronization sequences. Nevertheless, the GLRT2 receiver may be very costly to implement, for a large number of antennas in particular, since for a $(M \times N)$ MIMO system, it requires both an $(N \times N)$ matrix inversion and an $(N \times N)$ or $(M \times M)$ determinant computation at each tested sample position. An alternative to the GLRT2 receiver is the MMSE receiver proposed in [6]. However, the MMSE receiver is shown in this paper to be very sensitive to training sequence correlation, which may limit its practical use in this context.

As complexity is an important issue for practical implementations, we propose several ways to decrease the complexity of the GLRT2 receiver while retaining its performance. The first way concerns MIMO systems with $M=2$ transmit antennas and consists in computing explicitly the determinant appearing in the GLRT2 receiver formulation, allowing in particular a direct comparison with both the MMSE test proposed in [6] and SIMO receivers. As the direct computation of the determinant is complicated for $M>2$, a second proposition to decrease receiver complexity consists in introducing two new low-complexity MIMO receivers which are robust to interference. These two new receivers, called E0-GLRT3 and E1-GLRT3, correspond to two estimates of the GLRT receiver in known, stationary, Gaussian, spatially correlated and temporally white total noise, called GLRT3 receiver. For stationary interference, a third way to decrease the receiver complexity is to compute and inverse at a lower rate the data correlation matrix appearing in the receiver expressions by computing it on an observation interval greater than the synchronization sequence length. Finally, a fourth way to decrease the receiver complexity is to optimize the number of transmit antennas used for synchronization for a given value of the number of receive antennas and for given 
kinds of propagation channels. Note that such a problem has been preliminarily investigated in [4] in the DS-CDMA context and in [8] for precoded synchronization schemes. The performance of the proposed optimization schemes and associated receivers, jointly with their complexity, are analyzed in this paper and compared with that of the GLRT2 receiver, enlightening the practical interest of the former.

\section{MODEL AND PROBLEM FORMULATION}

\subsection{Hypotheses}

We consider a $(M \times N)$ MIMO radiocommunication link with $M$ and $N$ narrow-band antennas at transmission and reception respectively, and denote by $\mathbf{s}(k)$ the $(M \times 1)$ synchronization sequence vector at time $k$. Assuming a flat fading propagation channel and perfect (symbol-level) time and frequency synchronization, the vector, $\mathbf{x}(k)$ of the complex envelopes of the signals at the output of the $N$ receive antennas at time $k$ can be written as

$$
\mathbf{x}(k)=\mathbf{H s}(k)+\mathbf{v}(k)
$$

Here, $\mathbf{H}$ is the $(N \times M)$ channel matrix and $\mathbf{v}(k)$ the sampled total noise vector, which contains the potential contribution of MUI interferences, jammers and background noise and which is assumed to be uncorrelated with all the signals $\mathbf{s}(k)$. We assume that the $\mathbf{v}(k)$ are zero-mean, stationary, i.i.d, temporally white, circular, Gaussian samples with covariance matrix $\mathbf{R}=\mathbb{E}\left[\mathbf{v}(k) \mathbf{v}(k)^{H}\right]$, where $\mathrm{H}$ stands for conjugate transpose. Denoting by $\mathbf{X}$ and $\mathbf{V}$ the $(N \times K)$ observation and total noise matrices $\mathbf{X}=[\mathbf{x}(1), \ldots, \mathbf{x}(K)]$ and $\mathbf{V}=[\mathbf{v}(1), \ldots, \mathbf{v}(K)]$, and by $\mathbf{S}$ the $(M \times K)$ synchronization sequence matrix $\mathbf{S}=[\mathbf{s}(1), \ldots, \mathbf{s}(K)]$, we obtain

$$
\mathbf{X}=\mathbf{H S}+\mathbf{V}
$$

Note that the flat fading assumption is required to develop receivers with a limited complexity but is not required in practice where the considered receivers may be used even for frequency selective fading channels, considering multiple paths as interferences.

\subsection{Problem formulation}

The problem of time synchronisation of a MIMO link may be viewed as a detection problem with two hypotheses [6]. The first hypothesis $\left(\mathrm{H}_{1}\right)$ is that the signal matrix $\mathbf{S}$ is perfectly aligned in time with the observation matrix $\mathbf{X}$, and corresponds to model (2). The second hypothesis $\left(\mathrm{H}_{0}\right)$ is that there is no signal in the observation matrix $\mathbf{X}$, and corresponds to model (3) given by

$$
\mathbf{X}=\mathbf{V}
$$

The problem of synchronization then consists in elaborating a statistical test, function of the observations $\mathbf{X}$, and in comparing the value of this test to a threshold. If the threshold is exceeded, detection is considered. The performance of a synchronization test statistic is characterized by the probability of a correct detection under $\mathrm{H}_{1}\left(\mathrm{P}_{\mathrm{D}}\right)$ for a given false alarm probability $\left(\mathrm{P}_{\mathrm{FA}}\right)$, corresponding to the probability of exceeding the threshold under $\mathrm{H}_{0}$. Note that in practice, $\mathbf{X}$ contains the $K$ last received observation samples and is updated at each sample until the main detection.

\section{RECEIVERS IN THE LITERATURE}

In this section, we briefly recall the GLRT2 and MMSE receivers introduced in [6].

\subsection{GLRT2 receiver}

According to the Neyman-Pearson theory of detection, the optimal statistical test for the detection of matrix $\mathbf{S}$ from matrix $\mathbf{X}$ is the likelihood ratio test (LRT), which consists in comparing the function $L R T \triangleq p_{H_{1}}(\mathbf{X} \mid \mathbf{S}, \mathbf{H}, \mathbf{R}) / p_{H_{0}}(\mathbf{X} \mid \mathbf{R})$ to a threshold, where $p_{H_{i}}(\mathbf{X} \mid \ldots)(i=0,1)$ is the conditional probability density of $\mathbf{X}$ under $\mathrm{H}_{i}$. The expression of the LRT then takes the form

$$
\mathrm{LRT}=\frac{\prod_{k=1}^{K} p_{H_{1}}(\mathbf{v}(k) \mid \mathbf{s}(k), \mathbf{H}, \mathbf{R})}{\prod_{k=1}^{K} p_{H_{0}}(\mathbf{v}(k) \mid \mathbf{R})} .
$$

For our model, we have $p_{H_{1}}(\mathbf{v}(k) \mid \mathbf{s}(k), \mathbf{H}, \mathbf{R})=$ $\frac{1}{\pi^{N} \operatorname{det}(\mathbf{R})} e^{-(\mathbf{x}(k)-\mathbf{H s}(k))^{H} \mathbf{R}^{-1}(\mathbf{x}(k)-\mathbf{H s}(k))}$, and $p_{H_{0}}(\mathbf{v}(k) \mid \mathbf{R})=\frac{1}{\pi^{N} \operatorname{det}(\mathbf{R})} e^{-\mathbf{x}(k)^{H} \mathbf{R}^{-1} \mathbf{x}(k)}$ where $\operatorname{det}(\mathbf{R})$ means determinant of $\mathbf{R}$. As, in practice, $\mathbf{R}$ and $\mathbf{H}$ are unknown, they have to be replaced in (4) by their maximum likelihood (ML) estimates under each of the two hypotheses $\mathrm{H}_{1}$ and $\mathrm{H}_{0}$ (for $\mathbf{R}$ ), giving rise to the GLRT2 statistic. In these conditions, it has been shown in [6] that a sufficient statistic for the GLRT2 is given by

$$
\text { GLRT2 }=\operatorname{det}\left[\mathbf{I}_{K}-\hat{\mathbf{P}}_{s} \hat{\mathbf{P}}_{x}\right]^{-K}
$$

where $\hat{\mathbf{P}}_{s}$ and $\hat{\mathbf{P}}_{x}$ are $(K \times K)$ matrices corresponding to the projection operators onto the row spaces spanned by $\mathbf{S}$ and $\mathbf{X}$ respectively, defined by $\hat{\mathbf{P}}_{s} \triangleq \mathbf{S}^{H}\left(\mathbf{S S}^{H}\right)^{-1} \mathbf{S}$ and $\hat{\mathbf{P}}_{x} \triangleq$ $\mathbf{X}^{H}\left(\mathbf{X X}^{H}\right)^{-1} \mathbf{X}$. Defining the matrices $\mathbf{R}_{s s} \triangleq \mathbf{S S}^{H} / K$, $\hat{\mathbf{R}}_{x x} \triangleq \mathbf{X X}^{H} / K$ and $\hat{\mathbf{R}}_{x s} \triangleq \mathbf{X S}^{H} / K$ and using the properties of the determinant, it is straighforward to show that (5) can also be written as

$$
\begin{aligned}
\operatorname{GLRT} 2 & =\operatorname{det}\left[\mathbf{I}_{N}-\hat{\mathbf{R}}_{x x}^{-1} \hat{\mathbf{R}}_{x s} \mathbf{R}_{s s}^{-1} \hat{\mathbf{R}}_{x s}^{H}\right]^{-K} \\
& =\operatorname{det}\left[\mathbf{I}_{M}-\mathbf{R}_{s s}^{-1} \hat{\mathbf{R}}_{x s}^{H} \hat{\mathbf{R}}_{x x}^{-1} \hat{\mathbf{R}}_{x s}\right]^{-K}
\end{aligned}
$$

Expression (6), not presented in [6], requires that $\mathbf{R}_{s s}$ is invertible, which is assumed in the following. This is only possible if $M \leq K$, which is then assumed in the following. Under these assumptions, expressions (5), (6) show that, at each tested sample position, the GLRT2 receiver requires the computation of at least an $(N \times N)$ matrix inversion, $\hat{\mathbf{R}}_{x x}^{-1}$, and the determinant of a $(P \times P)$ matrix where $P=\operatorname{Inf}(K, N, M)$, which may be prohibitive for a large $K$ and a large number of antennas.

In the particular case of a SIMO system $(M=1), \hat{\mathbf{R}}_{x s}$ reduces to the vector $\hat{\mathbf{r}}_{x s_{1}}, \mathbf{R}_{s s}$ reduces to the scalar $r_{s_{1}}$ and 
we deduce from (6) that a sufficient statistic for the GLRT2 is given by

\subsection{MMSE receiver}

$$
\text { GLRT2 } 2_{S I M O}=\frac{\hat{\mathbf{r}}_{x s_{1}}^{H} \hat{\mathbf{R}}_{x x}^{-1} \hat{\mathbf{r}}_{x s_{1}}}{r_{s_{1}}} .
$$

MMSE time synchronization consists in finding the sample position which minimizes the least square (LS) error, $\hat{\epsilon}$, between the known sampled vectors $\mathbf{s}(k)$ and their LS estimation from a spatial filtering of the data $\mathrm{x}(k)(1 \leq k \leq K)$. After elementary computations, we obtain a sufficient statistic for the MMSE receiver, given by [6]

$$
\mathrm{MMSE} \triangleq \frac{\operatorname{Tr}\left(\hat{\mathbf{R}}_{x s}^{H} \hat{\mathbf{R}}_{x x}^{-1} \hat{\mathbf{R}}_{x s}\right)}{\operatorname{Tr}\left(\mathbf{R}_{s s}\right)}=\frac{\sum_{i=1}^{M} \hat{\mathbf{r}}_{x s_{i}}^{H} \hat{\mathbf{R}}_{x x}^{-1} \hat{\mathbf{r}}_{x s_{i}}}{\sum_{m=1}^{M} r_{s m}}
$$

where $\operatorname{Tr}()$ means Trace, $\hat{\mathbf{r}}_{x s_{i}}$ corresponds to the column $i$ of $\hat{\mathbf{R}}_{x s}$ and $r_{s m}$ is the term $(m, m)$ of $\mathbf{R}_{s s}$. Comparing (8) to (7), we deduce that, to within a constant, the MMSE receiver corresponds to the weighted sum of $M$ SIMO receivers, each of them associated with a particular transmit antenna. The computation of the MMSE receiver requires a $(N \times N)$ matrix inversion at each tested sample position but no determinant computation, which is less complex than the GLRT2 computation. For SIMO links $(M=1)$, the MMSE and GLRT2 receivers coincide, but this is a priori no longer true for $M>1$ as will be shown in the next section.

\section{DIRECT EXPRESSION OF GLRT2 FOR M=2}

For $M=2$, the determinant (6) can be easily computed and the result allows a direct comparison with the MMSE receiver (8). After straightforward computations of (6) for $M=2$, we obtain an alternative sufficient statistic for the GLRT2 as

$$
\begin{aligned}
& \operatorname{GLRT} 2=\hat{\mathbf{h}}_{1}^{H} \hat{\mathbf{R}}_{x x}^{-1} \hat{\mathbf{r}}_{x s_{1}}-\left(\hat{\mathbf{h}}_{1}^{H} \hat{\mathbf{R}}_{x x}^{-1} \hat{\mathbf{r}}_{x s_{1}}\right)\left(\hat{\mathbf{h}}_{2}^{H} \hat{\mathbf{R}}_{x x}^{-1} \hat{\mathbf{r}}_{x s_{2}}\right) \\
& \quad+\hat{\mathbf{h}}_{2}^{H} \hat{\mathbf{R}}_{x x}^{-1} \hat{\mathbf{r}}_{x s_{2}}+\left(\hat{\mathbf{h}}_{1}^{H} \hat{\mathbf{R}}_{x x}^{-1} \hat{\mathbf{r}}_{x s_{2}}\right)\left(\hat{\mathbf{h}}_{2}^{H} \hat{\mathbf{R}}_{x x}^{-1} \hat{\mathbf{r}}_{x s_{1}}\right)
\end{aligned}
$$

where $\hat{\mathbf{H}}=\left[\hat{\mathbf{h}}_{1}, \hat{\mathbf{h}}_{2}\right]$ is the ML estimate of $\mathbf{H}$ under $\mathrm{H}_{1}$, given by $\hat{\mathbf{H}}=\hat{\mathbf{R}}_{x s} \mathbf{R}_{s s}^{-1}$. For orthogonal sequences, we deduce that $\hat{\mathbf{h}}_{i}=r_{s_{i}}^{-1} \hat{\mathbf{r}}_{x s_{i}}(1 \leq i \leq 2)$, and comparing (9) with (8) for $M=2$, we deduce that for MIMO links $(M>1)$, the MMSE receiver no longer corresponds to the GLRT2 receiver, even for orthogonal synchronization sequences having the same power, which was not obvious a priori.

\section{NEW LOW-COMPLEXITY RECEIVERS}

The direct computation of the determinant (6) is not so straigthforward for $M>2$ while the MMSE receiver (8) has been shown in [6] to become sub-optimal for non-orthogonal synchronization sequences. In this context, a second way to decrease the complexity of the GLRT2 receiver for arbitrary values of $M$ while trying to keep its performance is to develop alternative receivers. To this aim, it seems natural to think that non-GLRT receivers corresponding to good estimates of the GLRT receiver in known total noise, called GLRT3 receiver, have good chances of approaching the performance of the GLRT2 receiver. For this reason, in this section, we introduce the GLRT3 receiver and propose two new low-complexity receivers corresponding to two different estimates of this receiver.

\subsection{GLRT3 receiver}

The GLRT3 receiver is obtained by considering expression (4) assuming an unknown channel matrix $\mathbf{H}$ and a zero-mean, i.i.d stationary, circular, Gaussian total noise whose covariance matrix, $\mathbf{R}$, is assumed to be known. Replacing in (4) $\mathbf{H}$ by its ML estimate $\hat{\mathbf{H}}=\hat{\mathbf{R}}_{x s} \hat{\mathbf{R}}_{s s}^{-1}$, valid for any $M$, generates the GLRT3 receiver. It is straightforward to show that a sufficient statistic for the GLRT3 receiver is given by

$$
\text { GLRT3 }=\operatorname{Tr}\left[\mathbf{R}_{s s}^{-1} \hat{\mathbf{R}}_{x s}^{H} \mathbf{R}^{-1} \hat{\mathbf{R}}_{x s}\right] \text {. }
$$

The GLRT3 receiver does not require any determinant computation, but unfortunately it cannot be used in practice since $\mathbf{R}$ is unknown. It can however be estimated by replacing $\mathbf{R}$ by an estimate, which is done in the following sections.

\subsection{Estimated GLRT3 receiver under $H_{0}$}

A first possibility to build from (10) a low-complexity receiver useful in practice is to replace in (10) the matrix $\mathbf{R}$ by its ML estimate under $\mathrm{H}_{0}, \hat{\mathbf{R}}_{0}$. It is easy to show that $\hat{\mathbf{R}}_{0}=\hat{\mathbf{R}}_{x x}$, which gives rise to the estimated GLRT3 receiver under $\mathrm{H}_{0}$ (E0-GLRT3), defined by

$$
\text { E0-GLRT3 }=\operatorname{Tr}\left[\mathbf{R}_{s s}^{-1} \hat{\mathbf{R}}_{x s}^{H} \hat{\mathbf{R}}_{x x}^{-1} \hat{\mathbf{R}}_{x s}\right] \text {. }
$$

For orthogonal sequences and to within a constant, (11) corresponds to (8). In this case, the MMSE receiver can be interpreted as an estimate of the GLRT3 receiver under $\mathrm{H}_{0}$. Otherwise, the MMSE receiver has no link with E0-GLRT3.

\subsection{Estimated GLRT3 receiver under $H_{1}$}

A second possibility to build from (10) a low-complexity receiver useful in practice is to replace in (10) the matrix $\mathbf{R}$ by its ML estimate under $\mathrm{H}_{1}, \hat{\mathbf{R}}_{1}$. It is easy to show that $\hat{\mathbf{R}}_{1}$ is defined by $\hat{\mathbf{R}}_{1}=\hat{\mathbf{R}}_{x x}-\hat{\mathbf{R}}_{x s} \mathbf{R}_{s s}^{-1} \hat{\mathbf{R}}_{x s}^{H}$. In $\hat{\mathbf{R}}_{1}$ the estimated contributions of the transmitted synchronization sequences have been removed from $\hat{\mathbf{R}}_{x x}$. This gives rise to the estimated GLRT3 receiver under $\mathrm{H}_{1}$ (E1-GLRT3), defined by

$$
\mathrm{E} 1-\mathrm{GLRT} 3=\operatorname{Tr}\left[\mathbf{R}_{s s}^{-1} \hat{\mathbf{R}}_{x s}^{H} \hat{\mathbf{R}}_{1}^{-1} \hat{\mathbf{R}}_{x s}\right] .
$$

\subsection{Performance of the estimated GLRT3 receivers}

To compare the performance of E0-GLRT3 and E1-GLRT3 with that of GLRT2, we consider a $(M \times N)$ MIMO link with omnidirectional transmitting and receiving antennas. The coefficients $\mathbf{H}_{i, j}(1 \leq i \leq N),(1 \leq j \leq M)$ of $\mathbf{H}$ are assumed to be zero-mean i.i.d circular Gaussian variables such that $\mathbb{E}\left[\left|\mathbf{H}_{i, j}\right|^{2}\right]=1$, which modelizes a Rayleigh flat fading model with a maximal diversity. The total noise is assumed to be composed of one single antenna interference and a background noise and is such that $\mathbf{v}(k)=j_{I}(k) \mathbf{h}_{I}+\mathbf{n}(k)$. 


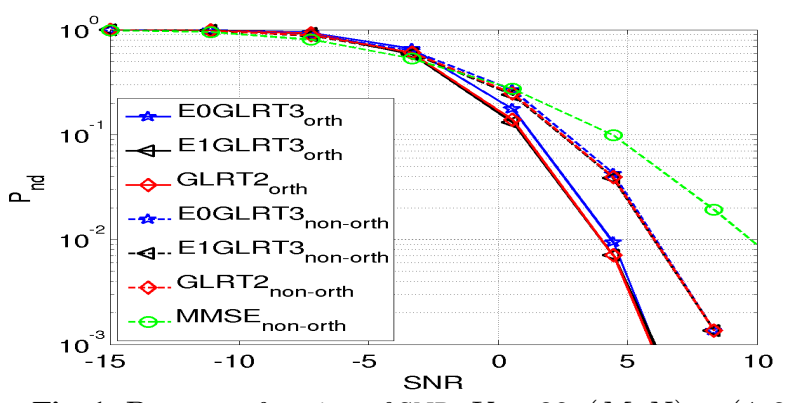

Fig. 1. $\mathrm{P}_{\mathrm{ND}}$ as a function of $S N R, K=32,(M, N)=(4,2)$

Here, $\mathbf{n}(k)$ is the sampled background noise vector, assumed to be zero-mean, stationary, Gaussian, SO circular, spatially white with a mean power per received antenna equal to $\eta_{2}$ and $\mathbf{h}_{I}$ the channel vector of the interference such that the components $\mathbf{h}_{I}[i](1 \leq i \leq N)$ are zero-mean i.i.d circular Gaussian variables verifying $\mathbb{E}\left[\left|\mathbf{h}_{I}[i]\right|^{2}\right]=1 . j_{I}(k)$ are the complex samples of the interference, assumed to be QPSK and such that $\pi_{I} \triangleq \mathbb{E}\left[\left|j_{I}(k)\right|^{2}\right]$ is the input mean power of the interference per antenna. Each synchronization sequence is composed of $K$ QPSK samples. The synchronization sequences have the same power $\left(r_{s_{i}} \triangleq r_{s}, 1 \leq i \leq M\right)$ and are normalized such that the signal to thermal noise ratio per receive antenna, defined by $\mathrm{SNR} \triangleq M r_{s} / \eta_{2}$, may be arbitrarily chosen. The interference to noise ratio per receive antenna, defined by INR $\triangleq \pi_{I} / \eta_{2}$, is chosen such that INR/SNR $=15$ $\mathrm{dB}$. The false alarm rate is $\mathrm{P}_{\mathrm{FA}}=10^{-3}$ and the figures are built from $10^{5}$ independent realizations.

Under these assumptions, Figures 1 and 2 show, for $K=$ 32 , for $(M, N)=(4,2),(4,4)$ respectively and for orthogonal and non-orthogonal synchronization sequences, the variations of the non-detection probability $\left(\mathrm{P}_{\mathrm{ND}}=1-\mathrm{P}_{\mathrm{D}}\right)$ as a function of the SNR per receive antenna at the output of the GLRT2, MMSE, E0-GLRT3 and E1-GLRT3 receivers. For non-orthogonal sequences, we repeat the same symbols in the beginning of the synchronization sequence for all antennas, such that $\left|\left(\rho_{s}\right)_{m, n}\right| \triangleq\left|\left(\mathbf{R}_{s s}\right)_{m, n}\right| /\left[r_{s n} r_{s m}\right]^{1 / 2}$ is comprised between 0.6 and $0.9(1 \leq m, n \leq M ; m \neq n)$. Figure 1 and 2 show an increasing performance of all the receivers with both the SNR and $N$, despite the presence of a strong interference, and decreasing performance of all the receivers with the correlation of the synchronization sequences. Moreover, these figures show very similar performances of E1-GLRT3 and GLRT2 and quite similar performance of E0-GLRT3 and GLRT2 whatever $(M, N)$, SNR and the correlation of the sequences. These results enlighten the practical interest of E0GLRT3 and E1-GLRT3 for any correlation between the sequence. Figures 1 and 2 show, for non-orthogonal sequences and SNR $>0 \mathrm{~dB}$, a degradation of the MMSE receiver with respect to the others, which is even more pronounced when $M$ is large compared with $N$. This illustrates the sub-optimality of this receiver for non-orthogonal sequences.

\section{COMPUTATION RATE DECREASE OF $\hat{\mathbf{R}}_{x x}$}

At each tested sample position, the use of GLRT2, MMSE, E0-GLRT3 and E1-GLRT3 requires the computation of both

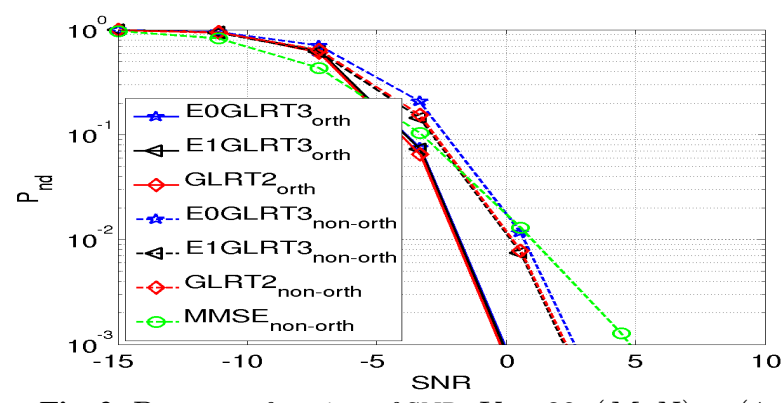

Fig. 2. $\mathrm{P}_{\mathrm{ND}}$ as a function of $S N R, K=32,(M, N)=(4,4)$

the $(N \times N) \hat{\mathbf{R}}_{x x}$ matrix over $K$ observation samples, and a $(N \times N)$ matrix inversion, which may become very costly for high values of $N$. In this context, a third way of decreasing the complexity of GLRT2 and E0-GLRT3 is to decrease the computation rate of $\hat{\mathbf{R}}_{x x}$ and its inverse by a factor $\beta>1$. The principle is to build an $\left(N \times K^{\prime}\right)$ observation matrix $\mathbf{X}^{\prime}=\left[\mathbf{x}(1), \ldots, \mathbf{x}\left(K^{\prime}\right)\right]$ from $K^{\prime}$ observation samples such that $K^{\prime}>K$ and, for the $\beta=K^{\prime}-K+1$ tested sample positions $l(1 \leq l \leq \beta)$, to replace in GLRT2 and E0-GLRT3 the $\hat{\mathbf{R}}_{x x}$ matrix by the $\hat{\mathbf{R}}_{x x}^{\prime}$ matrix defined by $\hat{\mathbf{R}}_{x x}^{\prime}=\mathbf{X}^{\prime} \mathbf{X}^{\prime H} / K^{\prime}$. Note that $K^{\prime}-K$ samples are now data samples instead of synchronization samples. As the data samples associated with different antennas are uncorrelated, this strategy to decrease the complexity of GLRT2 and E0-GLRT3 is only valid for orthogonal synchronization sequences. Further, this strategy requires constant values of $\mathbf{H}$ and $\mathbf{R}$ over $K^{\prime}$ samples, which may limit the value of $K^{\prime}$. However, it allows to compute and to inverse only one $(N \times N)$ matrix per set of $\beta$ tested sample positions, hence a gain of $\beta$ in the matrix inversion, while the matrix computation cost for the set of $\beta$ positions remains the same. Note that this strategy cannot be applied to E1-GLRT3 since the computation of $\hat{\mathbf{R}}_{1}$ requires an update of $\hat{\mathbf{R}}_{x s}$ at each time sample. Under the same assumptions as Figure 1, Figure 3 shows, for $(M, N)=(4,4)$ and $(2,8), K^{\prime} / K=2$ and 10 , the variations of $\mathrm{P}_{\mathrm{ND}}$ as a function of the SNR per receive antenna at the output of GLRT2, GLRT2-CRD and E0-GLRT3-CRD, where R-CRD means receiver $\mathrm{R}$ with a computation rate decrease. Note an increasing performance degradation of GLRT2-CRD and E0- GLRT3CRD with respect to GLRT2 (equivalent in this case to E0GLRT3) as $K^{\prime}$ / $K$ increases, while remaining lower than 1dB for $K^{\prime} / K=2$, which enlightens the interest of GLRT2-CRD and E0-GLRT3-CRD. Note also a better performance of E0GLRT3-CRD with respect to GLRT2-CRD for $K^{\prime} / K=10$, showing a better robustness of the former.

\section{OPTIMIZATION OF M}

As the complexity of all the previous receivers increases with the number of transmit antennas $M$, it is important in practice to wonder whether this parameter can be optimized for synchronization purposes. To this aim, let us note that, for a given value of $N$ and at least for high SNR, increasing $M$ while transmitting the same global power, should increase the spatial diversity order of the MIMO system for fading channels. However, increasing $M$ also increases the number of 


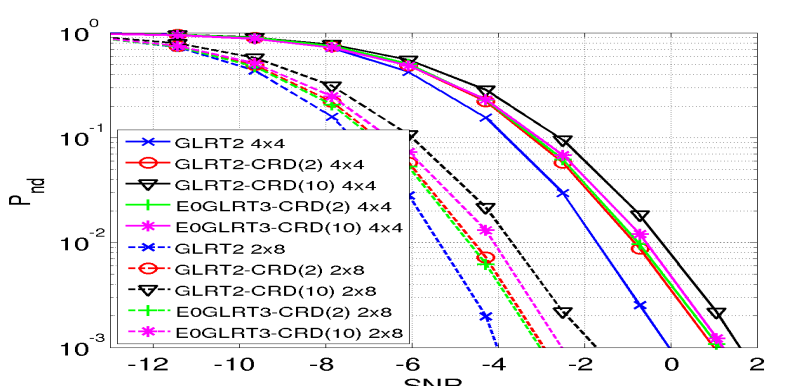

Fig. 3. CRD receivers, $(M, N) \stackrel{\text { SNR }}{=}(4,4),(2,8), K^{\prime} / K=2$ and 10 .

transmitted sequences and thus the amount of interference at reception. A compromise between diversity and interferences should then be found. To get new insights into this compromise, Figure 4 shows, for INR/SNR $=15 \mathrm{~dB}$, Rayleigh fading channels, orthogonal sequences of $K=32$ QPSK samples, $N=2$ and different values of $M$, the variations of $\mathrm{P}_{\mathrm{ND}}$ as a function of the SNR per receive antenna at the output of GLRT2. Similar results are obtained for E0-GLRT3 and E1GLRT3. At low SNR, the SIMO scheme is optimal for synchronization, proving in this case that the dominant limitation parameter are the interferences. On the contrary at high SNR, we note the sub-optimality of the SIMO scheme due to fading and increasing performance with $M$ as long as $M \leq 4$, due to an increase of the system diversity order up to 8 , a value for which the fading has practically disappeared. For $M>4$, i.e. above a system diversity order of 8 , the increase in the diversity gain is weak while the interference level increases, hence the non-increasing or even decreasing performance with increasing $M$. Similar results are obtained for $N>2$.

\section{COMPLEXITY ANALYSIS}

To get more insights into the relative complexity of the considered receivers, Figure 5 shows, for $K=32, K^{\prime} / K=10$, $M=2$ and $M=8$, the number of complex operations required by GLRT2, GLRT2-CRD, E0-GLRT2 and E0-GLRT3$\mathrm{CRD}$ as a function of $N$. Note the increasing polynomial complexity with $M$ and $N$ of all the receivers and the great interest of optimizing $M$ for both performance and complexity reasons. Note the lower complexities of receivers with CRD and the lowest complexity of E0-GLRT3-CRD. Note finally the lower complexity of E0-GLRT3 with respect to GLRT2 as $M$ increases.

\section{CONCLUSION}

In this paper, new insights into the time synchronization of $(M \times N)$ MIMO systems, corrupted by interferences of any

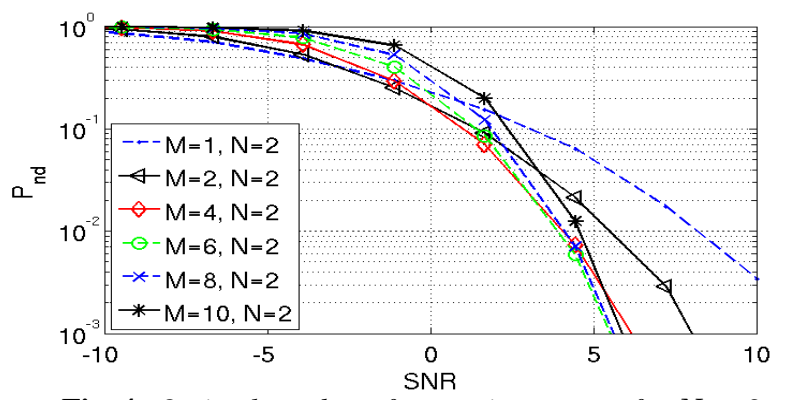

Fig. 4. Optimal number of transmit antennas for $N=2$.

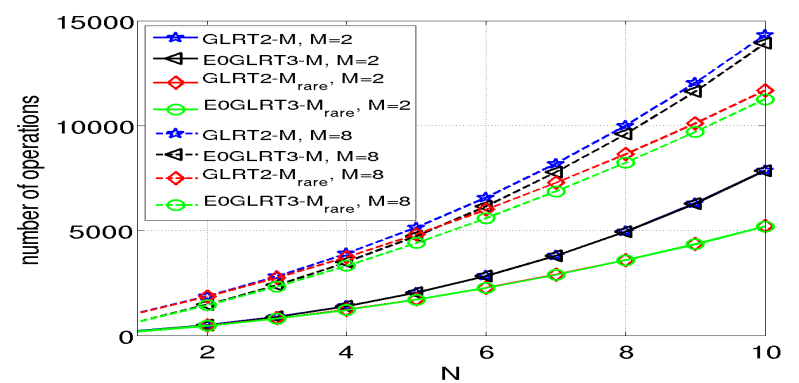

Fig. 5. Number of operations as a function of $N, K^{\prime}=10 K=320$.

kind, have been given, and several schemes aiming at reducing the complexity of GLRT2 presented in [6] have been proposed. Alternative expressions of GLRT2 have been introduced and the determinant computation has been done explicitly for $M=2$, allowing a direct comparison of GLRT2 with MMSE. Two new low-complexity receivers, E0-GLRT3 and E1-GLRT3, have been introduced, and have been shown to have a performance very close to that of GLRT2 for any correlation between sequences. A powerful procedure of computation rate reduction of the data correlation matrix has been proposed for orthogonal sequences. The problem of optimization of $M$ for time synchronization has been investigated for Rayleigh channels, showing, for high SNR, increasing performance with $M$ as long as $M N$ does not become much greater than 8. Finally, a complexity analysis confirms the practical interest of the proposals presented in this paper. All these results are useful for optimizing the choice and implementation of the receiver for time synchronization in practical systems.

\section{REFERENCES}

[1] A. Saemi, J. Cances, and V. Meghdadi, "Synchronization algorithms for mimo ofdma systems," IEEE Trans. Wireless. Commun., vol. 6, no. 12, pp. 4441-4451, 2007.

[2] M. Marey, O.A. Dobre, and R. Inkol, "A novel blind block timing and frequency synchronization algorithm for alamouti stbc," IEEE Comm. Letters, vol. 17, no. 3, pp. 569-572, 2013.

[3] Y. Wu, S. Chan, and E. Serpedin, "Symbol timing estimation in space-time coding systems based on orthogonal training sequences," IEEE Trans. On Wireless Communications, vol. 4, no. 2, pp. 603-613, 2005.

[4] S.H Won and L. Hanzo, "Non-coherent code acquisition in the multiple transmit/multiple receive antenna aided single- and multi-carrier ds-cdma downlink," IEEE Trans. On Wireless Communications, vol. 6, no. 11, pp. 3864-3869, 2007.

[5] T. Tang and R. Heath, "A space-time receiver with joint synchronization and interference cancellation in asynchronous mimo-ofdm systems," IEEE Trans. Vehicular Technology, vol. 57, no. 5, pp. 2991-3005, 2008.

[6] D. W. Bliss and P. A. Parker, "Temporal synchronization of mimo wireless communication in the presence of interference," IEEE Trans. Signal Process., vol. 58, no. 3, pp. 1794-1806, 2010.

[7] Y. Zhou, E.Serpedin, K. Qaraqe, and O. Dobre, "On the performance of generalized likelihood ratio test for data-aided timing synchronization of mimo systems," Proc. International Conf. on Communications (ICC'12), Bucharest, pp. 43-46, 2012.

[8] T. Koivisto and V. Koivunen, "Diversity transmission of synchronization sequences in mimo systems," IEEE Trans. On Wireless Comm., vol. 11, no. 11, pp. 4048-4057, 2012. 